\section{\$18. Disk Shaped Radiation Sources Fabricated by Compression and Formation of Sinter Powder}

Kawano, T.

Many materials contain naturally occurring radioisotopes, such as monazite, sinter (hot springs deposit), dried seaweed, fertilizer, and lantern mantles. These materials are often used as radiation samples in studies involving natural radioisotopes.

Recently, a method for the compression and formation of a radiation source with natural-radioisotope-containing materials has been developed. This method does not affect the amount and concentration of radioactivity in the material, despite reducing the volume of the original material. These materials are referred to as natural radiation sources. In the present study, a natural radiation source was fabricated by compression of sinter powder containing several radioisotopes of the uranium and thorium series.

The performance of the sinter radiation sources fabricated by the method was examined in radiation protection studies, which determined the dependence of radiation count rate on distance, shielding thickness, and shielding material. In these tests, the count rates were measured over 10 minutes with a GM survey meter.

For the distance dependence test, the distance between the source and probe was set from 0 to $30 \mathrm{~cm}$ for 11 sources and the count rates were obtained. A distance of zero means

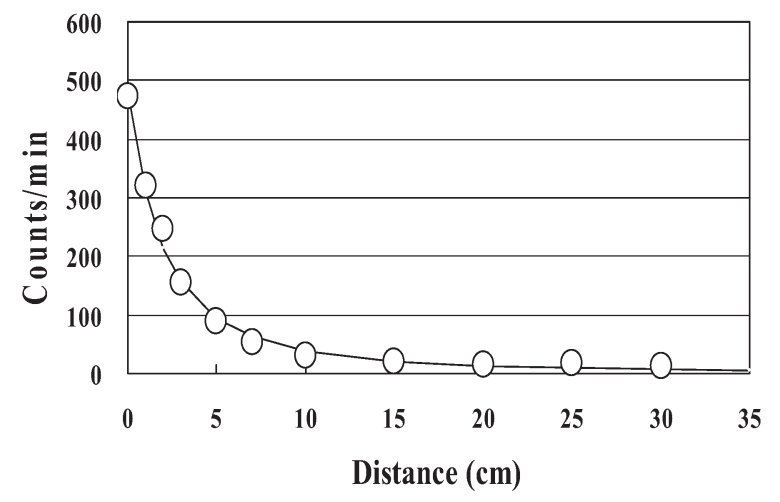

Fig.1 Dependence of radiation counts on distance.

that the source was directly in contact with the surface of the survey meter probe. Results are shown in Fig. 1 in which Xand Y-axes are distance and count rate, respectively. As the distance increased, the count rate decreased rapidly at first and then more moderately. This variation in count rate as a function of distance appeared to follow the inverse-square law.

To examine the dependence of radiation count rate on the material and its thickness, four types of thin plates were used as shielding materials. Only materials commonly available were selected as shielding to allow the experiments to be adapted for a basic radiation course appropriate for junior and senior high school students.

The distance between the probe and the sinter radiation source was fixed at $15 \mathrm{~mm}$, and various thicknesses of shielding materials were placed between the probe and source. Using a GM survey meter, the count rate was measured with and without shielding materials. By dividing the shielded count rates by unshielded count rates, respective transmission rates were obtained.

Figure 2 shows the transmission rate results, in which the $\mathrm{X}$ - and $\mathrm{Y}$-axes represent the thickness of shielding materials and transmission rate, respectively.

All transmission rates decreased exponentially with an increase in thickness in the order from greatest to lowest rate: copper $>$ aluminum $>$ plastic $>$ paper. Since mass

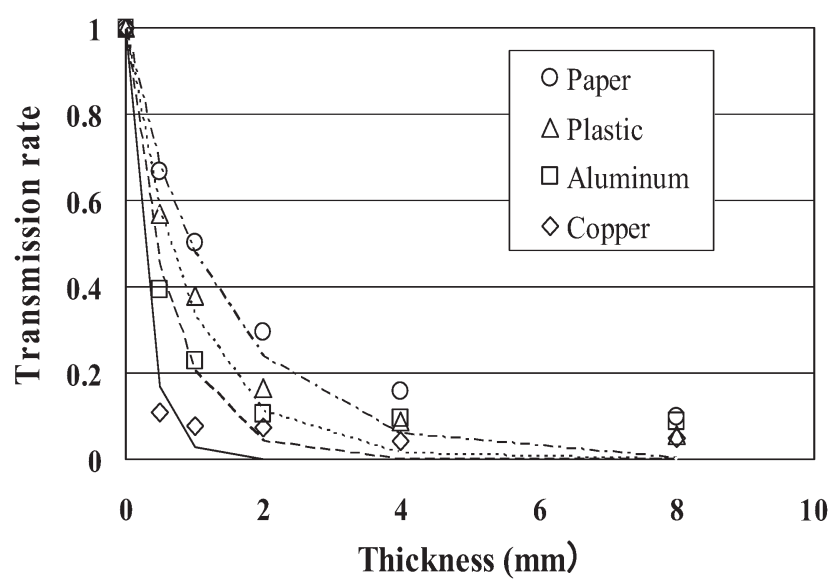

Fig.2 Dependence of radiation counts on shielding thickness of various materials.

densities of copper, aluminum, plastic, and paper are 8.9, 2.7, 1.35 , and $0.93 \mathrm{~g} / \mathrm{cm}^{3}$, respectively, the results explain the general principle of radiation shielding that materials of a larger mass density provide greater radiation shielding. This experiment explains, semi-quantitatively, the relationship between mass density and effectiveness of shielding against radiation.

The results showed that the sinter radiation source fabricated by compression and formation using sinter powder can be applied to demonstrate characteristics of radiation relating to distance, shielding thickness, and shielding material. The sinter radiation source is a natural substance, not a legal radioisotope, and thus can be safely and easily used in a basic course on radiation protection. This sinter radiation source is a useful teaching aid for courses in understanding radiation and its characteristics.

\section{Acknowledgement}

This work was supported by KAKENHI (Grant-in-Aid for Exploratory Research, 19650234). 